\title{
Intravenous penicillin: \\ the use of large doses in the treatment of endocarditis
}

\author{
HiLlas SMITH \\ M.D., M.R.C.P. \\ Senior Registrar, Royal Free Hospital, \\ Infectious Diseases Unit, Coppetts Wood Hospital, London, N.10
}

The Following case report which is presented in detail illustrates two important features of the treatment of complicated infections, namely, the possibility of giving large doses of penicillin intravenously for a long period without ill-effect, and also the difficulties which may arise in selecting an effective antibiotic regime.

\section{Case report}

B.G., a 36-year-old housewife, was admitted to the Unit in January 1964 with vaginal blood loss following a self-induced abortion. Prior to admission she was given oral penicillin for 2 days but the dose was not recorded. At the time of admission the patient was pale and anxious; she had lower abdominal tenderness and pelvic examination showed a widely dilated cervical canal with offensive retained products presenting. The heart was not enlarged; the first sound in the mitral area was intensified and the characteristic presystolic murmur of mitral stenosis was heard. The blood pressure was $110 / 80$.

The patient had a history of several episodes of rheumatic fever and chorea in childhood and had not been allowed to play games at school. From the age of 13 years she had not been kept under regular cardiac surveillance. She had had one previous pregnancy which terminated with a prolonged labour; the child was healthy.

After admission a course of 14.5 mega units penicillin intramuscularly with sulphatriad $42.5 \mathrm{~g}$ orally was given over a period of 8 days; on the 5 th day the uterus was evacuated under general anaesthesia. Culture from the cervix gave a growth of coliform organisms. Blood cultures were not made. $\mathrm{Hb} 75 \%$. White blood cell count $11,000 /$ $\mathrm{mm}^{3}$. Radiological examination of heart and lungs showed no abnormality and the electrocardiogram was within normal limits. The cardiac con- dition remained unchanged and no stigmata of endocarditis were observed. A temperature of $99.7^{\circ} \mathrm{F}$ was recorded 7 days after curettage but this subsided and the patient was discharged 4 days later, that is after a period of 16 days in hospital.

Seen 3 weeks later she reported she had frequency of micturition and dysuria and was found to be febrile. The presystolic murmur was unaltered. Microscopic examination of the urine showed many red blood cells, 30-35 white cells per $1 / 6$ field and coliform organisms, sensitive to chloramphenicol, were grown. WBC $6000 / \mathrm{mm}^{3}$. The patient was readmitted, a series of blood cultures was carried out and a course of $10 \mathrm{~g}$ chloramphenicol in 6 days was given for treatment of the urinary infection. A growth of Streptococcus faecalis was obtained from one blood culture; this organism was found to be sensitive to tetracycline, fucidin, oleandomycin, erythromycin and chloramphenicol but resistant to various antibiotics including penicillin and streptomycin. During the course of chloramphenicol therapy the temperature ranged from 99.7 to $101^{\circ} \mathrm{F}$ but the urinary symptoms abated and the urine became sterile. On the 8th day the tip of the spleen became palpable; at this time the temperature was $101^{\circ} \mathrm{F}$. The following day a course of fucidic acid was commenced. Two grams per day were given by the oral route. After 5 days' treatment there was no appreciable improvement and penicillin, 4 mega units i.m. daily, was added and the two drugs were continued for a period of 6 weeks. Pyrexia persisted through the first 4 weeks but in the last 2 the patient became apyrexial. At this time an aortic diastolic murmur was first noted. Within $24 \mathrm{hr}$ of discontinuing the drugs there was a recurrence of pyrexia and urinary symptoms. A series of blood cultures was done 
and Streptococcus faecalis type 9 was grown. This strain was referred to Professor Shooter, St Bartholomew's Hospital, for sensitivity assays. He reported that the strain was extremely resistant and that only a combination of penicillin, streptomycin and cepnaiosporin $\mathrm{C}$ in high concentration was bactericidal in vitro. Before this information became available a course of $1.6 \mathrm{~g}$ nitrofurantoin had been given over a period of 5 days. Beecham Laboratories had now reported that the original organism was sensitive to ampicillin.

On 27 April, 77 days after admission, the temperature was $99^{\circ} \mathrm{F}$, the spleen was easily palpable and an embolic node of the Osler type was noted on the right thumb. The following course of drugs was then commenced: penicillin 20 mega units and ampicillin $2 \mathrm{~g}$ daily intravenously, streptomycin $1 \mathrm{~g}$ intramuscularly and probenecid $2 \mathrm{~g}$ orally per diem. After 10 days cephalosporin C was added in doses of $3 \mathrm{~g}$ intramuscularly daily. After 16 days on this combination of drugs the patient continued to be febrile and there seemed to be no clinical response. On 13 May prednisolone, $30 \mathrm{mg}$ orally daily, was given and 3 days later ampicillin was discontinued after a total course of $34.5 \mathrm{~g}$. The same day the dose of penicillin was raised to 100 mega units intravenously daily; in addition the patient was now receiving streptomycin $1 \mathrm{~g}$ intramuscularly, cephalosporin $3 \mathrm{~g}$ intramuscularly, prednisolone $30 \mathrm{mg}$ and probenecid $1 \mathrm{~g}$ orally in each period of $24 \mathrm{hr}$. During the next 2 weeks a rise of temperature was recorded on only four occasions on the twice-daily chart. Serum titrated against the infecting organism was found to be bacteriostatic at a dilution of 1:64 and bactericidal at a dilution of $1: 4$.

The daily 100 mega unit dose of penicillin was given in 1.5 litres of $0.18 \%$ saline; the osmolality of the solution was found to be $283 \mathrm{mOsmol} / \mathrm{l}-$ very close to that of plasma. In each $24-\mathrm{hr}$ period the total quantity of sodium infused was 154 $\mathrm{mEq}$ and potassium $84 \mathrm{mEq}$ when the daily ration of penicillin was divided equally between the sodium and potassium salt and this was the combination most frequently used. After 5 weeks of intravenous therapy when various arm veins had been used, a catheter was inserted into the inferior vena cava via the femoral vein (Seldinger technique). The infusion then ran without trouble until the completion of intravenous therapy 2 months later.

After 7 weeks cephalosporin was discontinued as further supplies were not available. Streptomycin was withdrawn after a course of $80 \mathrm{~g}$ had been given. Penicillin therapy was stopped after 13 weeks but the catheter was left in situ in case

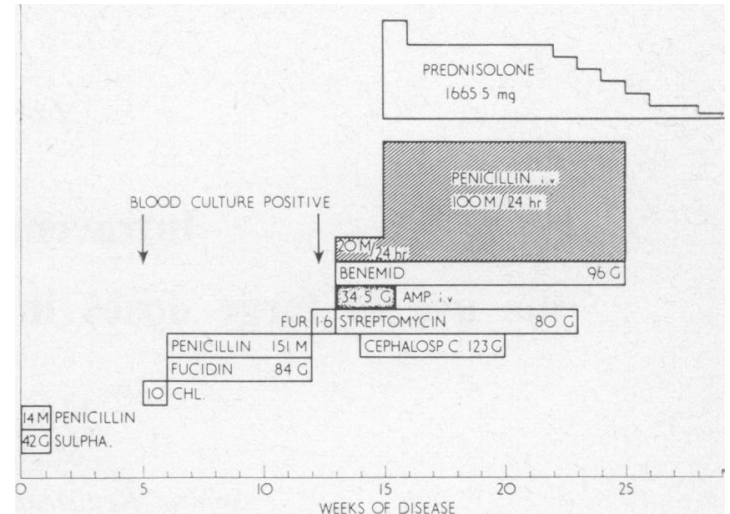

FIG. 1. Diagram showing dose and duration of various antibiotics.

of a return of pyrexia. Occasional peaks of temperature did occur especially during the 3-week period when corticosteroid drugs were withdrawn. During this time a vulval monilial infection was treated with nystatin and one tooth was extracted. The diagram shows the dose and duration of drugs prescribed.

Many blood cultures have now proved sterile; five urine cultures are also sterile. The electrocardiogram and chest film remain unchanged. Haemoglobin has risen to $87 \%$ and the ESR remains persistently below $10 \mathrm{~mm} / \mathrm{hr}$. The patient was discharged from hospital on 3 October 1964 after a period of $8 \frac{1}{2}$ months. She has remained well since that time; the mitral and aortic murmurs are unchanged. In March 1965 a complete dental clearance was carried out without complication.

\section{Discussion}

Although this patient had an infection of the heart valves with a highly resistant strain of Streptococcus faecalis the end result of treatment was not unfavourable. A charge of polypharmacy could certainly not be refuted but it must be conceded that initial antibiotic therapy was based on sensitivity patterns produced by routine laboratory investigation. The fact that the organism would only respond to a combination of penicillin, streptomycin and cephalosporin in high concentration in vitro fully justifies the use of high dosage of penicillin intravenously for a prolonged period and it is noteworthy that neither electrolyte imbalance, azotaemia nor evidence of neurotoxicity occurred. It is difficult to assess the part played by corticosteroids, which may have done little more than aid in the control of pyrexia; we formed the impression that they were helpful, they may on the other hand have contributed to 
the development of the moniliasis. It is unfortunate that serum and cerebrospinal fluid penicillin levels were not estimated.

\section{Summary}

A case of bacterial endocarditis due to a highly resistant strain of Streptococcus faecalis is described. Although some measure of control of infection was obtained by a combined course of fucidic acid and penicillin the condition relapsed immediately the drugs were discontinued. Finally, the intravenous administration of penicillin in doses of 100 mega units daily for 3 months in conjunction with streptomycin and cephalosporin C for shorter periods brought about satisfactory resolution without complications.

\section{Acknowledgments}

I wish to thank Dr A. M. Ramsay for permission to publish details of this patient who was under his care. Thanks are also due to Professor R. S. Shooter for antibiotic assays and also to Beecham and Glaxo Laboratories.

\title{
Transradial dorsal perilunate fracture dislocation: an unusual fracture dislocation of the wrist joint
}

\author{
C. Galasko* \\ M.B., B.Ch.(W.Rand), F.R.C.S., F.R.C.S.E. \\ Registrar, Johannesburg General Hospital. \\ Department of Orthopaedics, University of Witwatersrand
}

INJURIES to the wrist are common. Fracture of the scaphoid, dislocation of the lunate and dorsal perilunate dislocation of the carpus are problems seen frequently in a busy Accident Service.

The combination of a dorsal perilunate fracturedislocation of the wrist and a fractured radial styloid is a very unusual injury. We have not been able to find a similar case in the literature and therefore feel that this case is worth reporting.

\section{Case report}

A 52-year-old male was involved in a car accident. He was concussed for a short while after the accident and was not able to describe the mechanism of the injury. He was admitted to an outlying hospital, where no radiologic service was available over week-ends. He was treated for shock and his injured wrist was immobilized in an above-elbow cast. He was transferred to the Johannesburg General Hospital 72 hr later when his general condition was satisfactory.

Radiological examination at this stage revealed an avulsion fracture of the second cervical spine, a compression fracture of the sixth cervical vertebra and roentgenograms, taken through plaster, showed the presence of an unreduced transradial dorsal perilunate fracture-dislocation of the wrist (Figs. 1 and 2). There was a dorsal perilunate dislocation with a fracture of the radial styloid, the small fragment maintaining its relationship to the scaphoid. The next day, $96 \mathrm{hr}$

*Present address: Hammersmith Hospital, London, W12.

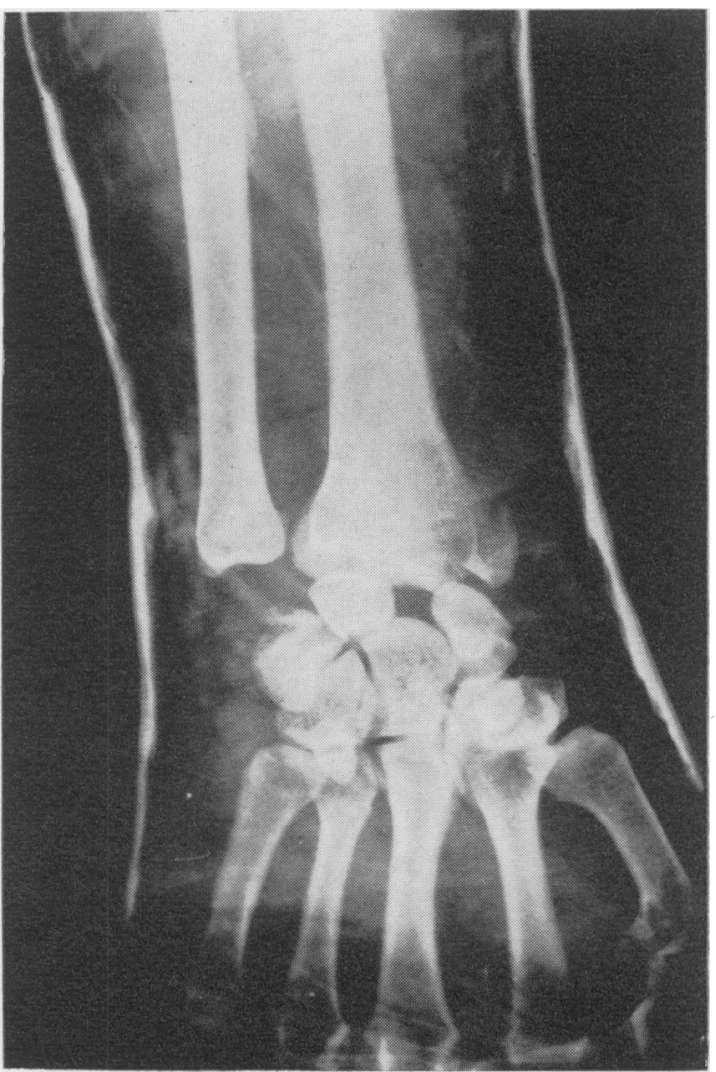

Fig. 1 . 